\title{
Editorial for the Introductory Issue of the Journal of Hardware and Systems Security (HaSS)
}

\author{
Swarup Bhunia $^{1} \cdot$ Mark Tehranipoor $^{1}$
}

Received: 22 May 2017 / Accepted: 24 May 2017 / Published online: 1 June 2017

(C) Springer 2017

It is our great pleasure to announce this inaugural issue of the Journal of Hardware and Systems Security (HaSS) by Springer. We believe it marks an important milestone as the first-ever journal on this very important topic area. The publication of the first issue of the journal is culmination of yearlong effort by the editor in chiefs, the distinguished editorial board members, and the Springer publication house.

The objective of this new journal is to provide unique coverage in an area of critical and rapidly growing significancenamely security and trust of electronic hardware and systems. It also aims at highlighting the role of hardware in security of computing and communication systems in the emergent internet of things (IoT) regime that is defined by an unprecedented scale of connected devices (or "things") with widely varied computing and sensing capabilities. The global economic trend that dictates a long and distributed design, fabrication, and distribution process of electronic hardware, coupled with their growing complexity and wide array of emerging applications - from smart cities to smart implants - is creating new hitherto unforeseen security/trust issues in the hardware "rootof-trust" as well as new demand for innovative effective countermeasures. Such a phenomenon has triggered vast widespread research activities in the domain of hardware system security and trust, leading to introduction of new conferences, new research programs by both federal agencies and industry, numerous special issues/sections in major journals, and series of new books by Springer and other major publishers. The number of publications in the field of hardware and system

Swarup Bhunia

swarup@ece.ufl.edu

1 University of Florida, Gainesville, FL, USA security is growing at a fast pace. Our analysis shows that the research activities in the diverse area of hardware and system security have gained significant momentum over the last decade, which is expected to be maintained in future years due to growing complexity of hardware, inevitable reliance on hardware when it comes to system security, and increasing application space of electronic hardware with enhanced connectivity and sensing.

Currently, there is no dedicated journal on hardware and system security. Publications in this area are scattered across many journals which often are not perfect fit within the scope of these journals. Hence, there was a critical need, expressed and shared by many fellow researchers and practitioners in this community, to introduce a dedicated journal on this topic from a reputed publishing house, like Springer, that provides a reliable venue for publishing high-quality research articles. Such a journal will be unique and comprehensive in coverage of topics. Hence, the initiative to create a new journal started with a strong motivation and encouragement from the community.

The journal is scheduled to be published quarterly (four issues in a calendar year), and in addition to the regular issues, the journal will periodically have special issues or sections dedicated to key topic areas or conferences/workshops on related topics. It plans to publish 6-10 peer-reviewed articles in each issue including regular as well as review/tutorial articles on key topic areas.

Specific topics that will be covered by HaSS include electronic hardware and system security encompassing all application domains, including embedded systems, cyber-physical systems, internet of things (IoT), reconfigurable (e.g., FPGA based) systems, and biomedical systems (e.g., implants, wearables). All abstraction levels of hardware-from hardware intellectual property (IP) blocks to microchips to printed circuit boards (PCBs) and systems - will be within the scope of 
HaSS journal. The interaction between hardware and firmware/software as it relates to system security and trust will also be covered by HaSS.

Intended audience of the journal includes: (1) researchers working in the broad area of electronic hardware and system security (embedded systems, cyber physical systems, biomedical systems, reconfigurable systems, and IoT) with focus on hardware security and trust at all levels of abstractions (IP, microchips, PCBs, and systems); (2) students (primarily from the area of computer engineering, electrical engineering, and computer science) interested in electronic hardware security; (3) security engineers and practitioners in electronics, semiconductor, and communication industry; and (4) federal employees/officials responsible for maintaining security/ integrity of government infrastructure.

The inaugural issue of HaSS contains the following six high-quality articles on diverse topics in hardware and system security. Technical contributions in these articles range from exploring new attack vector in processor architecture to new countermeasure for a critical attack on cryptosystem to benchmarking and security guidance for multitude of hardware trust issues. We are extremely happy to choose these articles which provide distinctive contribution in the field, for the inaugural issue from many submissions which are reviewed.

1. "Fault Tolerant Infective Countermeasure for AES", by S. Patranabis et al.

2. "Security Measures against a Rogue Network-on-Chip", by R. Jayashankara Shridevi et al.
3. "Security Assurance Guidance for Third-Party IP", by B. Sherman et al.

4. "Trust Filter: Runtime Hardware Trojan Detection in Behavioral MPSoCs", by N. Veeranna and B.C. Schafer

5. "Trace Buffer Attack on the AES Cipher", by Y. Huang et al.

6. "Benchmarking of Hardware Trojans and Maliciously Affected Circuits", B. Shakya et al.

We greatly appreciate the support from the hardware and system security community in response to this effort which has been both very encouraging and the driving force for us. We are grateful to the entire community for their positive response to establishing this new journal. We sincerely hope that you enjoy reading this inaugural issue as well as the future issues and engage in this effort in various roles - as authors, reviewers, editorial board members, and guest editors of special issues. We would like to thank all authors and reviewers for their tremendous efforts and contributions in producing these high-quality articles. We also take this opportunity to thank the editorial board and the entire staff in the Springer Journal Production Office, in particular to Marjorei Paran and Jim Langlois for their encouragement and assistance in delivering this issue. Finally, we express our heartfelt thanks and gratitude to Charles Glaser, Editorial Director at Springer, for taking us through the whole process of introducing this new journal with always helpful and valuable inputs.

Editor in Chiefs

Journal of Hardware and Systems Security (HASS) 